\title{
Study on Efficacy of Pesticide Mixtures as Foliar Sprays for the Management of Rice Leaf Folder (Cnaphalocrocis medinalis) and Leaf Blast (Pyricularia oryzae) on Paddy
}

\author{
K. Anil Kumar ${ }^{1 *}$, A.S.R. Sarma ${ }^{2}$, T. Madhumathi ${ }^{1}$ and V. Prasanna Kumari ${ }^{3}$ \\ ${ }^{1}$ Department of Entomology, Agricultural College, Bapatla \\ ${ }^{2}$ Department of Entomology, DAATTC, Peddapuram \\ ${ }^{3}$ Department of Plant Pathology, Agricultural College, Bapatla \\ *Corresponding author
}

\section{A B S T R A C T}

\section{Keywords}

Pesticide, Tank mix,

Leaf folder, Leaf blast

Article Info

Accepted:

15 October 2018

Available Online:

10 November 2018
A field experiment was conducted at Agricultural College Farm, Bapatla to test the efficacy of some recommended insecticides and fungicides as a tank mix application. Two insecticidal treatments (chlorantraniliprole @ $0.3 \mathrm{ml} \mathrm{l}^{-1}$ and chlorpyriphos@ $2.5 \mathrm{ml} \mathrm{l}^{-1}$ ) and two fungicidal treatments (tricyclazole @ $0.6 \mathrm{~g} \mathrm{l}^{-1}$ and isoprothiolane @ $1.5 \mathrm{ml} \mathrm{l}^{-1}$ ) with an untreated control were replicated thrice in a simple randomized block design. The results showed that all the insecticide and fungicide treatments alone and in combination were found superior over untreated check. Amongst them, chlorantraniliprole + isoprothiolane $\left(0.3 \mathrm{ml} \mathrm{l}^{-1}+1.5 \mathrm{ml} \mathrm{l}^{-1}\right)$ was the most effective treatment to control leaf folder infestation with 83.04 per cent leaf damage reduction over control and tricyclazole $\left(0.6 \mathrm{~g} \mathrm{l}^{-1}\right)$ alone was superior in reducing leaf blast severity with 74.13 per cent disease reduction over control.

\section{Introduction}

Rice (Oryza sativa L.), the staple food of $65 \%$ of the total population in India. In India, paddy is grown in $44.06 \mathrm{M}$ ha constituting $34.4 \%$ of the total cultivable area with production of 105.31 $\mathrm{M} \mathrm{t}$ and the productivity being $2178 \mathrm{t}$ $\mathrm{ha}^{-1}$.

In Andhra Pradesh, it occupied an area of 4.51 $\mathrm{M}$ ha with a production of $13.03 \mathrm{M} \mathrm{t}$ and productivity of $2891 \mathrm{~kg} \mathrm{ha}^{-1}$ during 2008-09 (Directorate of Economics and Statistics, 2013-14).
The rice leaf folder, Cnaphalocrocis medinalis (Guenee) earlier, was considered as a minor pest, but now has assumed major pest status in the entire country particularly in areas of high fertilizer usage. In conducive environment this pest may cause severe damage at maximum tillering and flowering stages of the crop which may lead to 60 to $70 \%$ leaf damage with $50 \%$ reduction in yield (Kushwana and Singh, 1984).

Among diseases, rice blast, bacterial blight, sheath blight and sheath rot are considered as major diseases because of their wide occurrence. Blast is a most serious disease of 
rice because of its devastating nature, faster spread, wider distribution and existence of several physiological races. Blast symptoms appear on leaves, nodes and grains. In general $40-50 \%$ reduction in yield was recorded but on severe incidence it varies from $70-80 \%$ (Prajapathi et al., 2004).

Insect-pests of rice like stem borer, leaf folder, diseases like blast and sheath blight coexist in rice ecosystem which, farmers have to manage simultaneously. Chemical control is one of the best and effective methods of pest control. It is the most commonly practicing method by farmers in rice that has made pest management highly complicated due to which farmers were forced to increase number of sprays, incurring additional cost. Considering these factors, a novel method called pesticide mixture has emerged where the compatible and effective insecticide and fungicide combinations were formulated and applied as a single tank mix which saves time, labour, energy, equipment cost to the farmers and prevents ecological problems like enhanced phytotoxicity, resurgence etc but Common growers find difficulty in ascertaining the compatibility of agrochemicals. Keeping these problems in view, a study was conducted to test the efficacy of insecticides and fungicides as tank mix application in paddy against rice leaf folder and leaf blast.

\section{Materials and Methods}

A field experiment was conducted during kharif, 2014 in the Agricultural College Farm, Bapatla to evaluate the efficacy of foliar sprays of insecticides, fungicides alone and in combination on leaf folder and leaf blast of paddy. BPT 5204 (Samba mashuri) was selected and laid out in a simple Randomised Block Design (RBD) with Nine treatments replicated thrice including the untreated check. Calculated quantities of selected insecticides and combinations were measured with the help of micro pipettes and mixed with required quantities of water to get desired dilution and sprayed with a hand compression knapsack sprayer. After every application of each of the treatments, the sprayer was thoroughly washed and rinsed twice with water and used for further treatments (Table $1)$.

\section{Data recording}

\section{Leaf folder (Cnaphalocrosis medinalis)}

The treatments were imposed when the population crossed the ETL, i.e., one larva per hill or one damaged leaf per hill. Observations on number of damaged leaves or average damaged leaves (ADL) on the randomly selected 20 per hill in each treatment under each replication were recorded at a day before spraying and on $5^{\text {th }}, 10^{\text {th }}$ and $15^{\text {th }}$ day after treatment imposition. Finally mean per cent leaf damage per hill was calculated from the data obtained by adopting the following formula.

Number of damaged leaves per hill

Per cent leaf folder infestation $=---------\times 100$

Total number of leaves per hill

\section{Leaf blast (Pyricularia oryzae)}

The data on blast was taken from five sampling units each of one square metre area in each plot at random and disease severity was recorded at 10 days interval from the day of its appearance and terminal severity at heading stage following 0-9 scale as per the SES (Standard Evaluation Scale). Per cent disease index was calculated and analyzed after suitable transformation.

\section{Yield}

The yield data was recorded by harvesting net plots replication wise excluding two border rows and yield per plot was recorded in $\mathrm{kg}$, 
based on which yield per hectare was calculated.

The yield data in each treatment was recorded separately and subjected to statistical analysis to test the significance of mean yield in different treatments. The per cent increase in yield over control in various treatments was calculated by using the following formula.

Per cent increase of yield in treatment over control $=[$ Yield in treatment - Yield in control / Yield in control] X 100

\section{Statistical Analysis}

Data on the leaf damage of leaf folder and per cent disease index of blast were transformed into angular/arc sine values and subjected to ANOVA in simple RBD analysis.

\section{Results and Discussion}

\section{Efficacy of different pesticides after first spray}

The results pertaining to efficacy of the treatments against per cent leaf damage by leaf folder on rice during first spray are presented in Table 2 and Figure 1.

The mean data on percent leaf damage of five, ten and fifteen days after spraying showed that chlorantraniliprole + isoprothiolane $\left(0.3 \mathrm{ml} \mathrm{l}^{-}\right.$ $\left.{ }^{1}+1.5 \mathrm{ml} \mathrm{l}^{-1}\right)$ is the best treatment by recording lowest (9.10) per cent leaf damage and was on par with chlorantraniliprole $\left(0.3 \mathrm{ml} \mathrm{l}^{-1}\right)$ with 9.48 per cent leaf damage.

Isoprothiolane $(1.5 \mathrm{ml} / \mathrm{l})$ and tricyclazole $(0.6$ $\mathrm{g} / \mathrm{l})$ recorded higher per cent leaf damage of 32.29, 35.09 per cent leaf damage respectively since, they have no insecticidal properties. However, untreated check recorded the highest per cent leaf damage of $32.08 \%$.

Efficacy of different pesticides after second spray

The results with regard to the efficacy of the treatments after second spray were presented in Table 3 and Figure 2.

The mean data at fifth, tenth and fifteenth day after second spraying revealed that chlorantraniliprole + isoprothiolane $\left(0.3 \mathrm{ml} \mathrm{l}^{-1}\right.$ $+1.5 \mathrm{ml} \mathrm{l}^{-1}$ ) was the best treatment by recording lowest (4.69) mean per cent leaf damage followed by chlorantraniliprole $(0.3$ $\mathrm{ml} \mathrm{l}^{-1}$ ) with 2.27 mean per cent leaf damage and chlorpyriphos $\left(2.5 \mathrm{ml} \mathrm{l}^{-1}\right)$ with 6.32 mean per cent leaf damage. However, untreated check recorded the highest per cent leaf damage of $45.70 \%$.

\section{Cumulative efficacy of different pesticides after two sprayings}

The data with regard to cumulative efficacy of the treatments with respect to per cent leaf damage are presented in Table 4 and Figure 3. The overall cumulative efficacy of the observations recorded at five, ten and fifteen days after two sprayings inferred that chlorantraniliprole + isoprothiolane $\left(0.3 \mathrm{ml} \mathrm{l}^{-1}\right.$ $+1.5 \mathrm{ml} \mathrm{l}^{-1}$ ) was the most effective treatment among all with 6.89 mean per cent leaf damage. These results are in corroboration with Bhuvaneswari and Krishnamraju (2013), who reported lower leaf folder incidence $(3.7 \%)$, lower stem borer incidence $(6.3 \%$ white ears), lower mean brown plant hopper population (20.01) with chlorantraniliprole $18.5 \% \mathrm{SC}+$ hexaconazole $5 \%$ EC. Chlorantraniliprole $\quad\left(\begin{array}{lll}0.3 & \mathrm{ml} & \left.\mathrm{l}^{-1}\right)\end{array}\right.$ and chlorpyriphos $\left(2.5 \mathrm{ml} \mathrm{l}^{-1}\right)$ are the next best treatments with 7.37 and 8.26 mean per cent leaf damage. These are in accordance with Suri (2011), who reported lower leaf folder damage $(3.40 \%)$ and stem borer damage (1.62\% dead hearts) with chlorantraniliprole @ $40 \mathrm{~g}$ a.i ha ${ }^{-1}$.

The results pertaining to the treatments clearly 
indicated that all the insecticidal treatments alone and in combination with fungicides were not significantly different from each other with respect to reduction of leaf folder incidence. However, these were significantly different from untreated control and fungicidal treatments.

\section{Efficacy of different pesticides against the rice leaf blast after spraying}

The symptoms of leaf blast appeared first at 25 days after transplantation (DAT) and the treatments were imposed at 35 DAT. The results pertaining to efficacy of the treatments against leaf blast incidence on rice are presented in Table 5 and Figure 4.

There was no significant difference between the different treatments at one day before spraying and per cent leaf blast severity ranged from 17.77 to 23.92 per 20 hills. 10 days after spray, the treatment, tricyclazole $\left(0.6 \mathrm{~g} \mathrm{l}^{-1}\right)$ was found as the best treatment by recording lowest (15.85) per cent leaf blast incidence which was on par with isoprothiolane $\quad\left(\begin{array}{lll}1.5 & \mathrm{ml} & \mathrm{l}^{-1}\end{array}\right)$ and chlorantraniliprole + isoprothiolane $\left(0.3 \mathrm{ml} \mathrm{l}^{-}\right.$ ${ }^{1}+1.5 \mathrm{ml} \mathrm{l}^{-1}$ ) which recorded 17.11 and $18.37 \%$ blast incidence, respectively. But, the treatments chlorpyriphos $\left(2.5 \mathrm{ml} \mathrm{l}^{-1}\right)$ and chlorantraniliprole $\left(0.3 \mathrm{ml} \mathrm{l}^{-1}\right)$ recorded higher per cent blast incidence of 30.22 and 32.81, respectively since they are insecticides without fungicidal action. However, untreated check recorded the highest per cent leaf blast incidence of $36.14 \%$. At 20 days after spraying (DAS), tricyclazole $\left(0.6 \mathrm{~g} \mathrm{l}^{-1}\right)$ found to be the best treatment by recording lowest (11.85) per cent leaf blast incidence which was on par with isoprothiolane $\left(1.5 \mathrm{ml} \mathrm{l}^{-1}\right)$ which recorded $13.48 \%$ leaf blast incidence followed by chlorantraniliprole + isoprothiolane $\left(0.3 \mathrm{ml} \mathrm{l}^{-1}+1.5 \mathrm{ml} \mathrm{l}^{-1}\right)$ which recorded $16.70 \%$ leaf blast. However, untreated check recorded the highest per cent leaf blast incidence of $41.92 \%$.

Fig.1 Efficacy of treatments (mean) after first spray against rice leaf folder Cnaphalocrosis medinalis $\mathrm{L}$.

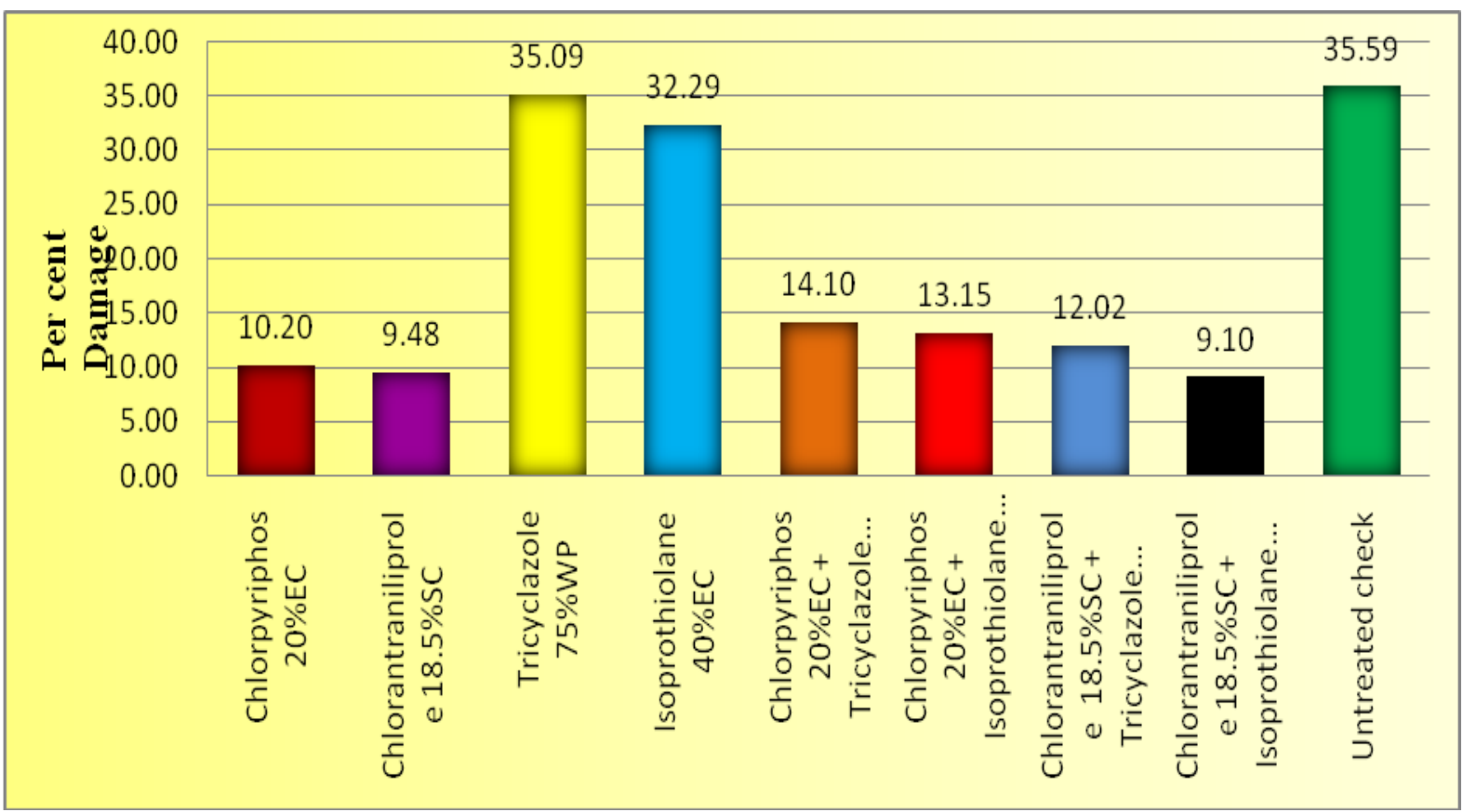


Fig.2 Efficacy of treatments (mean) after second spray against rice leaf folder Cnaphalocrosis medinalis L.

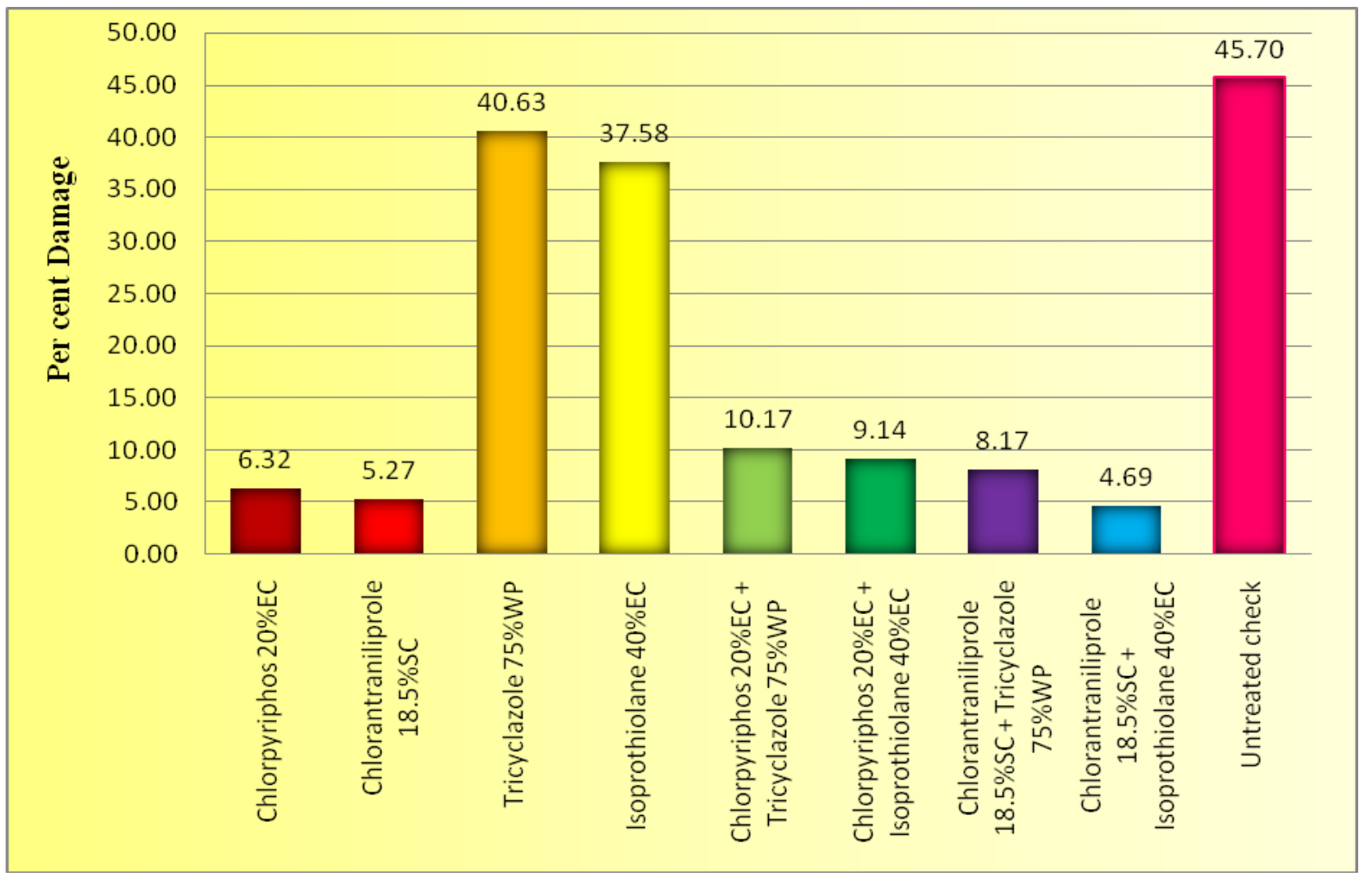

Fig.3 Efficacy of treatments (cumulative mean) against rice leaf folder Cnaphalocrosis medinalis L.

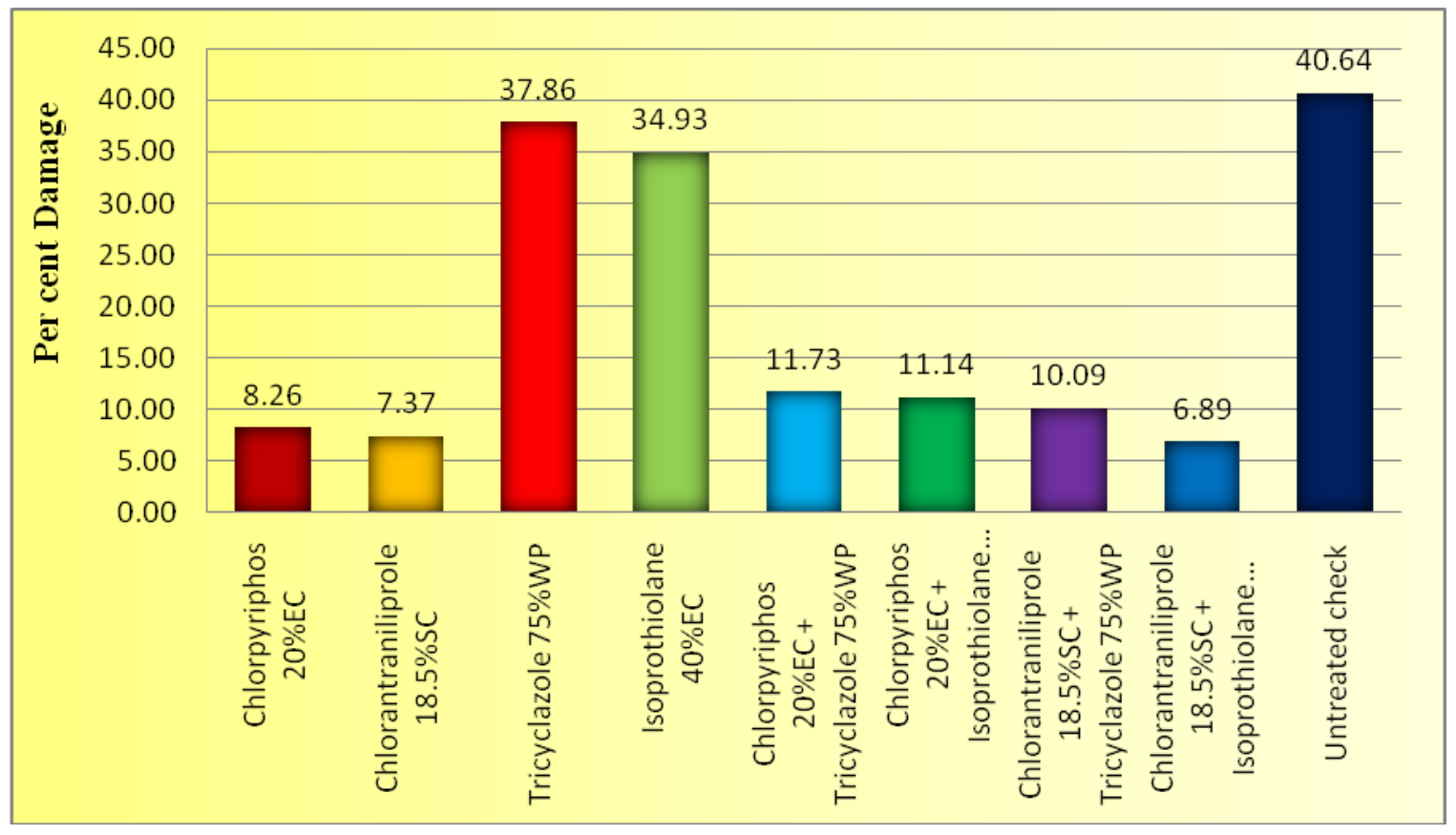


Fig.4 Efficacy of treatments (mean) against rice leaf blast, Pyricularia oryzae

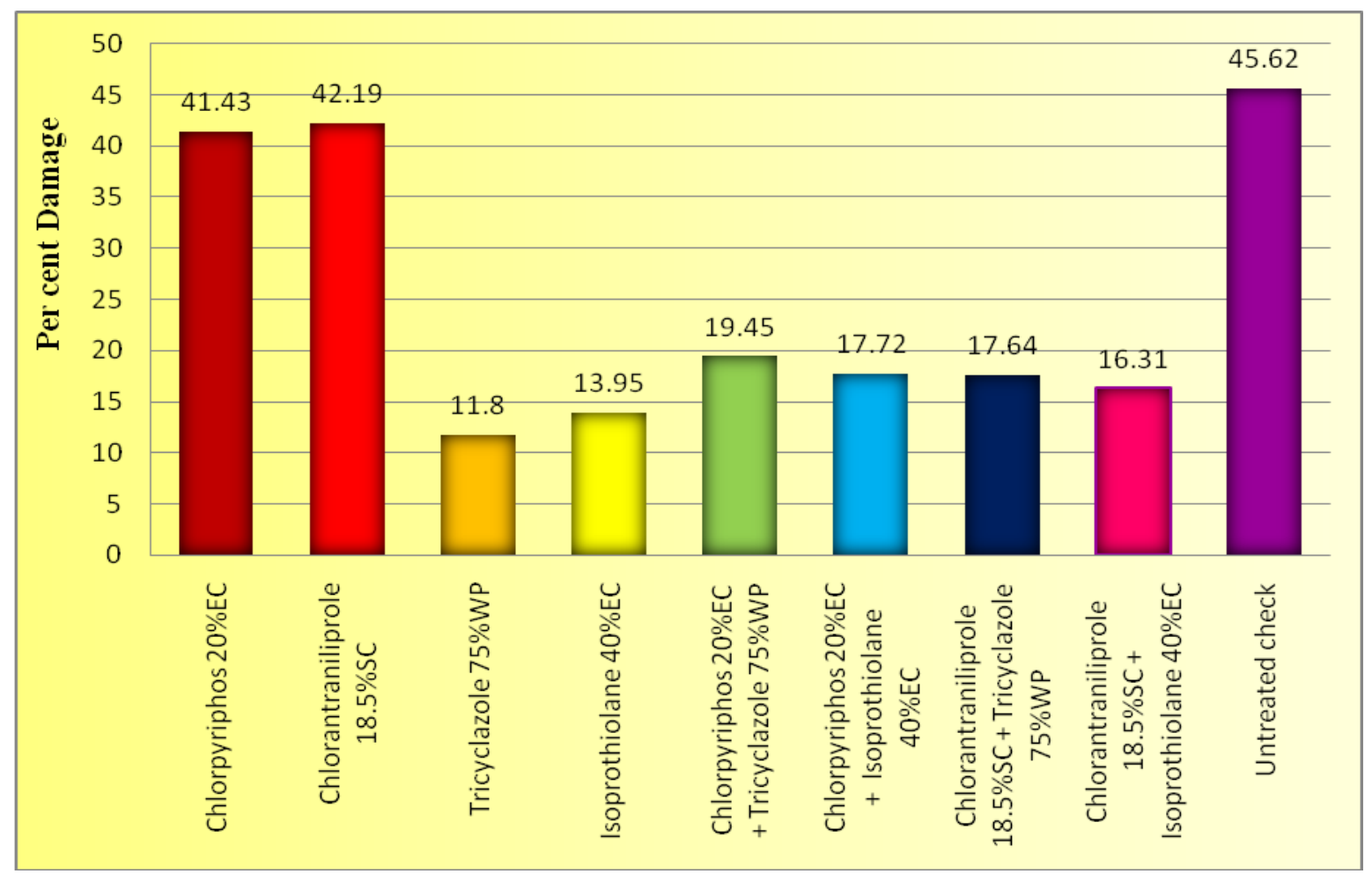

Fig.5 Efficacy of treatments on the yield of paddy

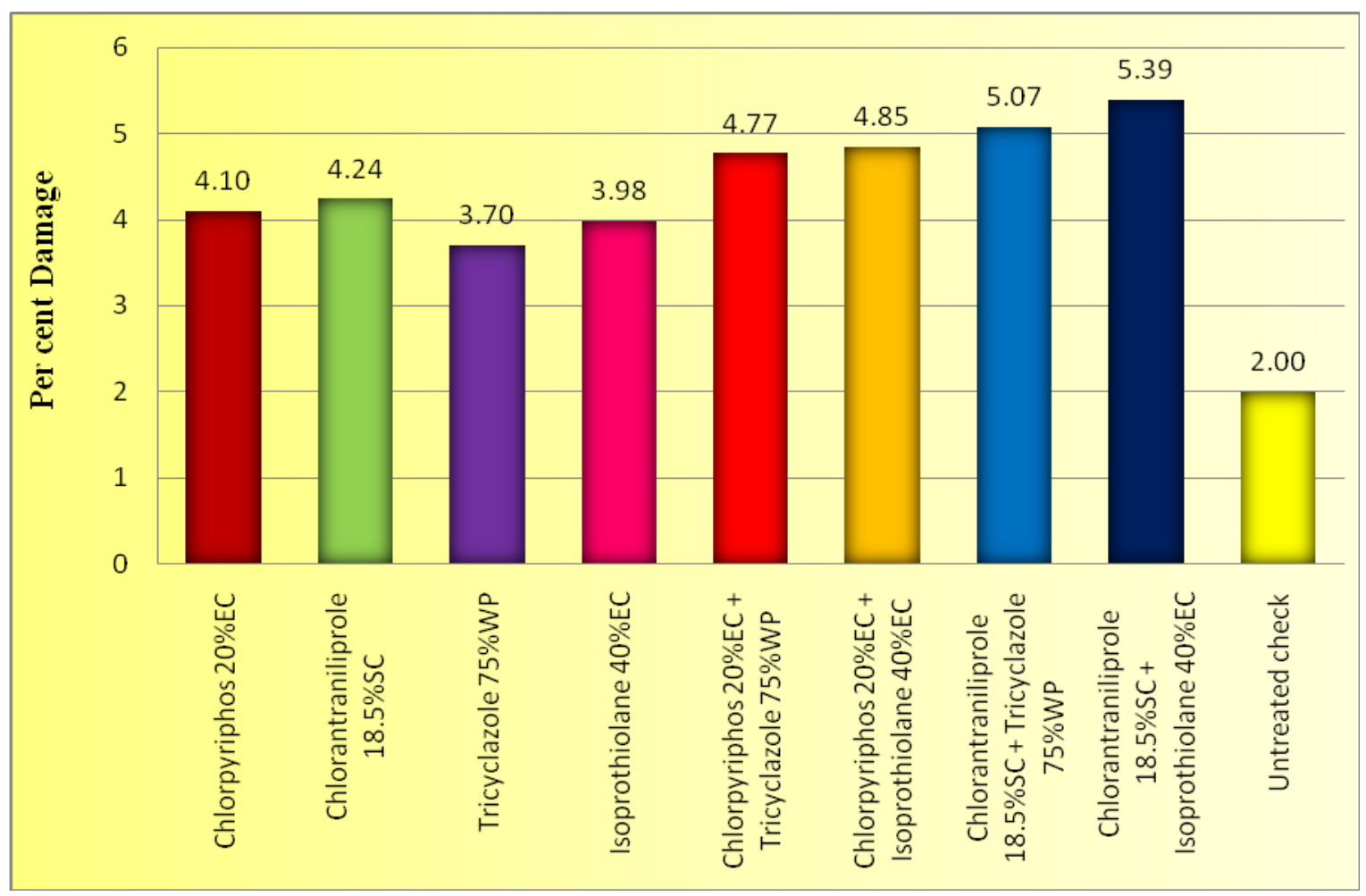


Table.1 Particulars of insecticides, fungicides alone and their combination Used in the experiment

\begin{tabular}{|c|c|c|}
\hline S. No. & Treatments (Trade name) & $\begin{array}{l}\text { Recommended } \\
\text { Concentration } \\
\left(\mathrm{ml} \text { or } \mathrm{g} \mathrm{l}^{-\mathbf{1}}\right)\end{array}$ \\
\hline 1. & Chlorpyriphos $20 \%$ EC (Classic) & 2.5 \\
\hline 2. & Chlorantraniliprole $18.5 \%$ SC (Coragen) & 0.3 \\
\hline 3. & Tricyclazole 75\% WP (Baan) & 0.6 \\
\hline 4. & Isoprothiolane $40 \% \mathrm{EC} \mathrm{(Fuzi-one)}$ & 1.5 \\
\hline 5. & Chlorpyriphos $20 \% \mathrm{EC}+$ tricyclazole $75 \% \mathrm{WP}$ & $2.5+0.6$ \\
\hline 6. & Chlorpyriphos $20 \%$ EC + isoprothiolane $40 \%$ EC & $2.5+1.5$ \\
\hline 7. & Chlorantraniliprole $18.5 \% \mathrm{SC}+$ tricyclazole $75 \% \mathrm{WP}$ & $0.3+0.6$ \\
\hline 8. & Chlorantraniliprole $18.5 \% \mathrm{SC}+$ isoprothiolane $40 \% \mathrm{EC}$ & $0.3+1.5$ \\
\hline 9. & Untreated control & - \\
\hline
\end{tabular}

Table.6 Efficacy of insecticides on yield of paddy

\begin{tabular}{|c|c|c|c|c|}
\hline $\begin{array}{l}\text { S. } \\
\text { No }\end{array}$ & Treatments & $\begin{array}{l}\text { Dose } \\
\left(\mathrm{ml} \text { or } \mathrm{g} \mathrm{l}^{-1}\right)\end{array}$ & $\begin{array}{l}\text { Yield } \\
\left(\mathrm{t} \mathrm{ha}^{-1}\right)\end{array}$ & $\begin{array}{l}\text { Increase over } \\
\text { control }(\%)\end{array}$ \\
\hline $\mathbf{T}_{1}$ & Chlorpyriphos $20 \%$ EC & 2.5 & $4.10^{\mathrm{bcd}}$ & 51.16 \\
\hline $\mathbf{T}_{2}$ & Chlorantraniliprole $18.5 \%$ SC & 0.3 & $4.24^{\mathrm{bcd}}$ & 52.71 \\
\hline$\overline{T_{3}}$ & Tricyclazole $75 \%$ WP & 0.6 & $3.70^{d}$ & 45.86 \\
\hline $\mathrm{T}_{4}$ & Isoprothiolane $40 \% \mathrm{EC}$ & 1.5 & $3.98^{\mathrm{cd}}$ & 49.61 \\
\hline $\mathbf{T}_{5}$ & $\begin{array}{l}\text { Chlorpyriphos } \quad 20 \% \quad \text { EC } \quad+ \\
\text { Tricyclazole } 75 \% \text { WP }\end{array}$ & $2.5+0.6$ & $4.77^{\mathrm{abc}}$ & 58.02 \\
\hline $\mathrm{T}_{6}$ & $\begin{array}{lr}\text { Chlorpyriphos } 20 \% \\
\text { Isoprothiolane } 40 \% \text { EC }\end{array}$ & $2.5+1.5$ & $4.85^{\mathrm{ab}}$ & 58.71 \\
\hline $\mathbf{T}_{7}$ & $\begin{array}{l}\text { Chlorantraniliprole } 18.5 \% \\
\text { Tricyclazole } 75 \% \text { WP }\end{array}$ & $0.3+0.6$ & $5.07^{\mathrm{a}}$ & 60.44 \\
\hline $\mathbf{T}_{8}$ & $\begin{array}{l}\text { Chlorantraniliprole } 18.5 \% \quad \mathrm{SC}+ \\
\text { Isoprothiolane } 40 \% \mathrm{EC}\end{array}$ & $0.3+1.5$ & $5.39^{\mathrm{a}}$ & 62.80 \\
\hline $\mathrm{T}_{9}$ & Untreated control & - & $2.00^{\mathrm{e}}$ & - \\
\hline & F test & & $\mathrm{S}$ & \\
\hline & SEm \pm & & 0.267 & \\
\hline & $\mathrm{CD}(\mathrm{P}=0.05)$ & & 0.80 & \\
\hline & $\mathrm{CV}(\%)$ & & 14.22 & \\
\hline
\end{tabular}

*Means followed by same letter do not differ significantly following DMRT 
Table.2 Efficacy of different pesticides against Rice Leaf folder, Cnaphalocrocis medinalis after first spray

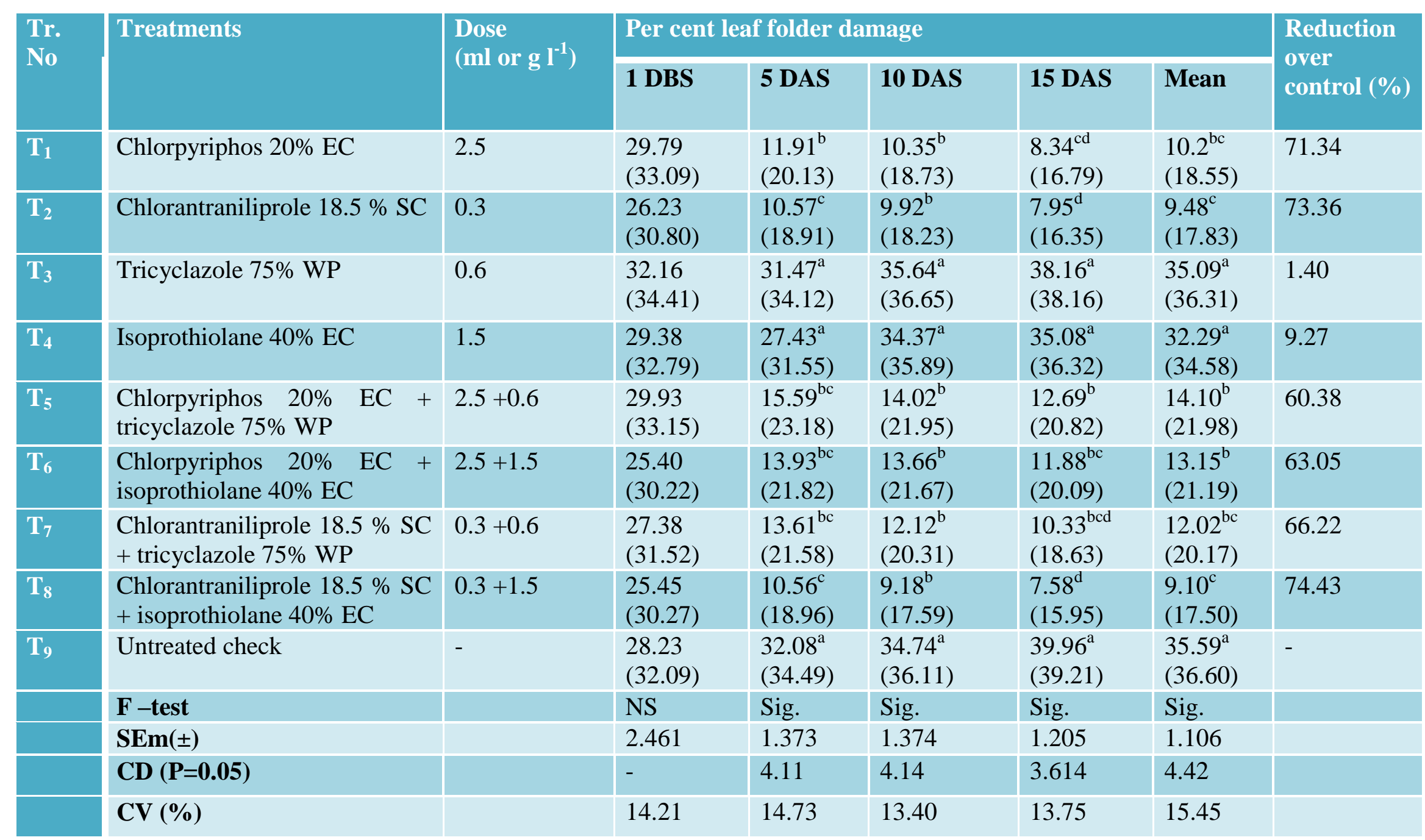

*DBS- day before spray $\quad * *$ DAS- days after spray

***Figures in parenthesis are Arc- sine transformed values

Means followed by same letter do not differ significantly following DMRT 
Table.3 Efficacy of different pesticides against Rice Leaf folder after second spray

\begin{tabular}{|c|c|c|c|c|c|c|c|c|}
\hline \multirow{2}{*}{$\begin{array}{l}\text { Tr. } \\
\text { No }\end{array}$} & \multirow[t]{2}{*}{ Treatments } & \multirow{2}{*}{$\begin{array}{l}\text { Dose } \\
(\mathrm{ml} \text { or } \mathrm{g} / \mathrm{l})\end{array}$} & \multicolumn{5}{|c|}{ Per cent leaf folder damage } & \multirow{2}{*}{$\begin{array}{l}\text { Reduction } \\
\text { over } \\
\text { control (\%) }\end{array}$} \\
\hline & & & $1 \mathrm{DBS}$ & 5 DAS & 10 DAS & 15 DAS & Mean & \\
\hline$\overline{T_{1}}$ & Chlorpyriphos $20 \%$ EC & 2.5 & $\begin{array}{l}39.30 \\
(38.81)\end{array}$ & $\begin{array}{l}8.32^{\mathrm{c}} \\
(16.70)\end{array}$ & $\begin{array}{l}6.64^{\mathrm{d}} \\
(14.83)\end{array}$ & $\begin{array}{l}4.02^{d} \\
(11.48)\end{array}$ & $\begin{array}{l}6.32^{\mathrm{de}} \\
(14.33)\end{array}$ & 86.10 \\
\hline $\mathbf{T}_{2}$ & Chlorantraniliprole $18.5 \%$ SC & 0.3 & $\begin{array}{l}38.47 \\
(38.31)\end{array}$ & $\begin{array}{l}7.23^{\mathrm{d}} \\
(15.57)\end{array}$ & $\begin{array}{l}4.86^{\mathrm{d}} \\
(12.69)\end{array}$ & $\begin{array}{l}3.72^{\mathrm{d}} \\
(11.03)\end{array}$ & $\begin{array}{l}5.27^{\mathrm{e}} \\
(13.09)\end{array}$ & 88.46 \\
\hline $\mathbf{T}_{\mathbf{3}}$ & Tricyclazole $75 \%$ WP & 0.6 & $\begin{array}{l}39.34 \\
(38.84)\end{array}$ & $\begin{array}{l}38.11^{\mathrm{b}} \\
(38.14)\end{array}$ & $\begin{array}{l}40.84^{\mathrm{ab}} \\
(39.74)\end{array}$ & $\begin{array}{l}42.95^{b} \\
(40.97)\end{array}$ & $\begin{array}{l}40.63^{a b} \\
(39.61)\end{array}$ & 11.09 \\
\hline $\mathbf{T}_{4}$ & Isoprothiolane $40 \% \mathrm{EC}$ & 1.5 & $\begin{array}{l}37.33 \\
(37.64)\end{array}$ & $\begin{array}{l}34.64^{\mathrm{b}} \\
(36.07)\end{array}$ & $\begin{array}{l}37.62^{b} \\
(37.85)\end{array}$ & $\begin{array}{l}40.50^{b} \\
(39.54)\end{array}$ & $\begin{array}{l}37.58^{b} \\
(37.82)\end{array}$ & 17.76 \\
\hline $\mathbf{T}_{5}$ & $\begin{array}{l}\text { Chlorpyriphos } 20 \% \\
\text { Tricyclazole } 75 \% \text { WP }\end{array}$ & $2.5+0.6$ & $\begin{array}{l}40.57 \\
(39.55)\end{array}$ & $\begin{array}{l}11.12^{\mathrm{c}} \\
(19.46)\end{array}$ & $\begin{array}{l}10.40^{c} \\
(18.78)\end{array}$ & $\begin{array}{l}8.99^{c} \\
(17.43)\end{array}$ & $\begin{array}{l}10.17^{\mathrm{c}} \\
(18.55)\end{array}$ & 77.74 \\
\hline$\overline{T_{6}}$ & $\begin{array}{l}\text { Chlorpyriphos } 20 \% \text { EC }+ \\
\text { Isoprothiolane } 40 \% \mathrm{EC}\end{array}$ & $2.5+1.5$ & $\begin{array}{l}40.97 \\
(39.78)\end{array}$ & $\begin{array}{l}11.43^{c} \\
(19.71)\end{array}$ & $\begin{array}{l}8.02^{\mathrm{cd}} \\
(16.42)\end{array}$ & $\begin{array}{l}8.01^{\mathrm{c}} \\
(16.40)\end{array}$ & $\begin{array}{l}9.14^{\mathrm{cd}} \\
(17.40)\end{array}$ & 80.00 \\
\hline $\mathbf{T}_{7}$ & $\begin{array}{l}\text { Chlorantraniliprole } 18.5 \% \mathrm{~S} \mathrm{C} \\
\text { + Tricyclazole } 75 \% \mathrm{WP}\end{array}$ & $0.3+0.6$ & $\begin{array}{l}39.22 \\
(38.76)\end{array}$ & $\begin{array}{l}9.30^{\mathrm{c}} \\
(17.70)\end{array}$ & $\begin{array}{l}8.31^{\mathrm{cd}} \\
(16.73)\end{array}$ & $\begin{array}{l}6.92^{\mathrm{cd}} \\
(15.23)\end{array}$ & $\begin{array}{l}8.17^{\mathrm{cd}} \\
(16.55)\end{array}$ & 82.12 \\
\hline$\overline{\mathrm{T}_{8}}$ & $\begin{array}{l}\text { Chlorantraniliprole } 18.5 \% \text { SC } \\
\text { + Isoprothiolane } 40 \% \mathrm{EC}\end{array}$ & $0.3+1.5$ & $\begin{array}{l}37.47 \\
(37.72)\end{array}$ & $\begin{array}{l}7.09^{\mathrm{d}} \\
(15.35)\end{array}$ & $\begin{array}{l}4.04^{\mathrm{d}} \\
(11.45)\end{array}$ & $\begin{array}{l}2.94^{\mathrm{d}} \\
(9.70)\end{array}$ & $\begin{array}{l}4.69^{e} \\
(12.16)\end{array}$ & 89.73 \\
\hline $\mathbf{T}_{9}$ & Untreated check & - & $\begin{array}{l}42.01 \\
(40.39)\end{array}$ & $\begin{array}{l}42.89^{\mathrm{a}} \\
(40.93)\end{array}$ & $\begin{array}{l}44.49^{\mathrm{a}} \\
(42.60)\end{array}$ & $\begin{array}{l}49.72^{\mathrm{a}} \\
(44.86)\end{array}$ & $\begin{array}{l}45.7^{\mathrm{a}} \\
(42.79)\end{array}$ & - \\
\hline & F test & & Sig. & Sig. & Sig. & Sig. & Sig. & \\
\hline & SEm \pm & & 2.25 & 1.02 & 1.13 & 1.119 & 1.251 & \\
\hline & $\mathrm{CD}(\mathrm{P}=0.05)$ & & NS & 3.062 & 3.390 & 3.356 & 3.605 & \\
\hline & CV (\%) & & 10.05 & 14.44 & 13.18 & 12.98 & 15.38 & \\
\hline
\end{tabular}

*DBS- day before spray $\quad * *$ DAS- days after spray

***Figures in parenthesis are Arc- sine transformed values 
Table.4 Cumulative Efficacy of different pesticides against Rice Leaf folder

\begin{tabular}{|c|c|c|c|c|c|c|c|c|}
\hline \multirow{2}{*}{$\begin{array}{l}\text { Tr. } \\
\text { No }\end{array}$} & \multirow[t]{2}{*}{ Treatments } & \multirow{2}{*}{$\begin{array}{l}\text { Dose } \\
(\mathrm{ml} \text { or } \mathrm{g} / \mathrm{l})\end{array}$} & \multicolumn{3}{|c|}{ Mean per cent damage } & \multicolumn{3}{|c|}{ Reduction over control } \\
\hline & & & $\begin{array}{l}\text { First } \\
\text { Spray }\end{array}$ & $\begin{array}{l}\text { Second } \\
\text { spray }\end{array}$ & $\begin{array}{l}\text { Cumulative } \\
\text { mean }\end{array}$ & $\begin{array}{l}\text { First } \\
\text { spray }\end{array}$ & $\begin{array}{l}\text { Second } \\
\text { spray }\end{array}$ & $\begin{array}{l}\text { Cumulative } \\
\text { Mean }\end{array}$ \\
\hline $\mathbf{T}_{1}$ & Chlorpyriphos $20 \% \mathrm{EC}$ & 2.5 & $\begin{array}{l}10.2^{\mathrm{bc}} \\
(18.55)\end{array}$ & $\begin{array}{l}6.32^{\mathrm{de}} \\
(14.33)\end{array}$ & $\begin{array}{l}8.26^{\text {cde }} \\
(16.44)\end{array}$ & 71.34 & 86.1 & 79.67 \\
\hline $\mathbf{T}_{2}$ & Chlorantraniliprole $18.5 \%$ SC & 0.3 & $\begin{array}{l}9.48^{c} \\
(17.83)\end{array}$ & $\begin{array}{l}5.27^{\mathrm{e}} \\
(13.09)\end{array}$ & $\begin{array}{l}7.37^{\mathrm{ef}} \\
(15.46)\end{array}$ & 73.36 & 88.46 & 81.86 \\
\hline $\mathbf{T}_{3}$ & Tricyclazole $75 \%$ WP & 0.6 & $\begin{array}{l}35.09^{\mathrm{a}} \\
(36.31)\end{array}$ & $\begin{array}{l}40.63^{a b} \\
(39.61)\end{array}$ & $\begin{array}{l}37.86^{\mathrm{ab}} \\
(37.96)\end{array}$ & 1.40 & 11.09 & 6.84 \\
\hline $\mathbf{T}_{4}$ & Isoprothiolane $40 \% \mathrm{EC}$ & 1.5 & $\begin{array}{l}32.29^{\mathrm{a}} \\
(34.58)\end{array}$ & $\begin{array}{l}37.58^{\mathrm{b}} \\
(37.82)\end{array}$ & $\begin{array}{l}34.93^{b} \\
(36.2)\end{array}$ & 9.27 & 17.76 & 14.05 \\
\hline$\overline{T_{5}}$ & $\begin{array}{l}\text { Chlorpyriphos } 20 \% \\
\text { Tricyclazole } 75 \% \mathrm{WP}\end{array}$ & $2.5+0.6$ & $\begin{array}{l}14.10^{b} \\
(21.98)\end{array}$ & $\begin{array}{l}10.17^{\mathrm{c}} \\
(18.55)\end{array}$ & $\begin{array}{l}11.73^{\mathrm{c}} \\
(19.92)\end{array}$ & 60.38 & 77.74 & 71.13 \\
\hline $\mathrm{T}_{6}$ & $\begin{array}{l}\text { Chlorpyriphos } 20 \% \quad \mathrm{EC}+ \\
\text { Isoprothiolane } 40 \% \mathrm{EC}\end{array}$ & $2.5+1.5$ & $\begin{array}{l}13.15^{b} \\
(21.19)\end{array}$ & $\begin{array}{l}9.14^{\mathrm{cd}} \\
(17.40)\end{array}$ & $\begin{array}{l}11.14^{\mathrm{cd}} \\
(19.29)\end{array}$ & 63.05 & 80 & 72.58 \\
\hline$\overline{T_{7}}$ & $\begin{array}{l}\text { Chlorantraniliprole } 18.5 \% \text { SC } \\
\text { + Tricyclazole } 75 \% \mathrm{WP}\end{array}$ & $0.3+0.6$ & $\begin{array}{l}12.02^{b c} \\
(20.17)\end{array}$ & $\begin{array}{l}8.17^{\mathrm{cd}} \\
(16.55)\end{array}$ & $\begin{array}{l}10.09^{\text {cde }} \\
(18.36)\end{array}$ & 66.22 & 82.12 & 75.17 \\
\hline $\mathbf{T}_{8}$ & $\begin{array}{l}\text { Chlorantraniliprole } 18.5 \% \text { SC } \\
\text { + Isoprothiolane } 40 \% \mathrm{EC}\end{array}$ & $0.3+1.5$ & $\begin{array}{l}9.10^{c} \\
(17.50)\end{array}$ & $\begin{array}{l}4.69^{\mathrm{e}} \\
(12.16)\end{array}$ & $\begin{array}{l}6.89^{\mathrm{f}} \\
(14.83)\end{array}$ & 74.43 & 89.73 & 83.04 \\
\hline T9 & Untreated check & - & $\begin{array}{l}35.59^{\mathrm{a}} \\
(36.60)\end{array}$ & $\begin{array}{l}45.7^{\mathrm{a}} \\
(42.79)\end{array}$ & $\begin{array}{l}40.64^{\mathrm{a}} \\
(39.69)\end{array}$ & - & - & - \\
\hline & F test & & Sig. & - & Sig. & & & \\
\hline & SEm \pm & & 1.106 & 1.251 & 1.086 & & & \\
\hline & $\mathrm{CD}(\mathrm{P}=0.05)$ & & 4.42 & 3.605 & 3.557 & & & \\
\hline & CV (\%) & & 15.45 & 15.38 & 15.03 & & & \\
\hline
\end{tabular}


Table.5 Efficacy of different pesticides against leaf blast Pyricularia oryzae

\begin{tabular}{|c|c|c|c|c|c|c|c|c|}
\hline \multirow{2}{*}{$\begin{array}{l}\text { Tr. } \\
\text { No }\end{array}$} & \multirow[t]{2}{*}{ Treatments } & \multirow{2}{*}{$\begin{array}{l}\text { Dose } \\
\left(\mathrm{ml} \mathrm{or} \mathrm{g} \mathrm{l}^{-1}\right)\end{array}$} & \multicolumn{5}{|c|}{ Blast disease severity (\%) } & \multirow{2}{*}{$\begin{array}{l}\text { Reduction } \\
\text { over } \\
\text { control }(\%)\end{array}$} \\
\hline & & & $1 \mathrm{DBS}$ & $10 \mathrm{DAS}$ & 20 DAS & 30 DAS & Mean & \\
\hline $\mathbf{T}_{1}$ & Chlorpyriphos $20 \%$ EC & 2.5 & $\begin{array}{l}17.77 \\
(24.89)\end{array}$ & $\begin{array}{l}30.22^{\mathrm{a}} \\
(33.34)\end{array}$ & $\begin{array}{l}39.26^{\mathrm{a}} \\
(38.79)\end{array}$ & $\begin{array}{l}54.81^{\mathrm{a}} \\
(47.79)\end{array}$ & $\begin{array}{l}41.43^{\mathrm{a}} \\
(39.97)\end{array}$ & 9.18 \\
\hline $\mathbf{T}_{2}$ & Chlorantraniliprole $18.5 \%$ SC & 0.3 & $\begin{array}{l}20.22 \\
(26.72)\end{array}$ & $\begin{array}{l}32.81^{\mathrm{a}} \\
(34.91)\end{array}$ & $\begin{array}{l}42.22^{a} \\
(40.54)\end{array}$ & $\begin{array}{l}51.56^{\mathrm{a}} \\
(45.92)\end{array}$ & $\begin{array}{l}42.19^{a} \\
(40.45)\end{array}$ & 7.51 \\
\hline $\mathbf{T}_{3}$ & Tricyclazole $75 \% \mathrm{WP}$ & 0.6 & $\begin{array}{l}18.15 \\
(25.20)\end{array}$ & $\begin{array}{l}15.85^{\mathrm{d}} \\
(23.42)\end{array}$ & $\begin{array}{l}11.85^{\mathrm{d}} \\
(20.05)\end{array}$ & $\begin{array}{l}7.70^{\mathrm{c}} \\
(16.06)\end{array}$ & $\begin{array}{l}11.8^{b} \\
(19.84)\end{array}$ & 74.13 \\
\hline $\mathbf{T}_{4}$ & Isoprothiolane $40 \% \mathrm{EC}$ & 1.5 & $\begin{array}{l}17.79 \\
(24.84)\end{array}$ & $\begin{array}{l}17.11^{\mathrm{cd}} \\
(24.44)\end{array}$ & $\begin{array}{l}13.48^{\mathrm{d}} \\
(21.52)\end{array}$ & $\begin{array}{l}10.59^{\mathrm{bc}} \\
(18.80)\end{array}$ & $\begin{array}{l}13.95^{b} \\
(21.58)\end{array}$ & 69.42 \\
\hline $\mathbf{T}_{5}$ & $\begin{array}{l}\text { Chlorpyriphos } 20 \% \quad \mathrm{EC} \quad+ \\
\text { Tricyclazole } 75 \% \mathrm{WP}\end{array}$ & $2.5+0.6$ & $\begin{array}{l}23.92 \\
(29.29)\end{array}$ & $\begin{array}{l}23.70^{\text {bc }} \\
(29.06)\end{array}$ & $\begin{array}{l}20.74^{b} \\
(27.06)\end{array}$ & $\begin{array}{l}13.93^{b} \\
(21.86)\end{array}$ & $\begin{array}{l}19.45^{b} \\
(25.99)\end{array}$ & 57.36 \\
\hline $\mathbf{T}_{6}$ & $\begin{array}{l}\text { Chlorpyriphos } 20 \% \quad \mathrm{EC}+ \\
\text { Isoprothiolane } 40 \% \mathrm{EC}\end{array}$ & $2.5+1.5$ & $\begin{array}{l}21.63 \\
(27.68)\end{array}$ & $\begin{array}{l}20.44^{\mathrm{c}} \\
(26.80)\end{array}$ & $\begin{array}{l}19.85^{\mathrm{bc}} \\
(26.42)\end{array}$ & $\begin{array}{l}11.70^{\mathrm{bc}} \\
(19.94)\end{array}$ & $\begin{array}{l}17.72^{b} \\
(24.38)\end{array}$ & 61.15 \\
\hline $\mathbf{T}_{7}$ & $\begin{array}{l}\text { Chlorantraniliprole } 18.5 \% \text { SC } \\
+ \text { Tricyclazole } 75 \% \mathrm{WP}\end{array}$ & $0.3+0.6$ & $\begin{array}{l}20.41 \\
(26.80)\end{array}$ & $\begin{array}{l}20.26^{c} \\
(26.69)\end{array}$ & $\begin{array}{l}22.07^{\mathrm{b}} \\
(28.03)\end{array}$ & $\begin{array}{l}10.61^{\mathrm{bc}} \\
(18.96)\end{array}$ & $\begin{array}{l}17.64^{b} \\
(24.56)\end{array}$ & 61.33 \\
\hline $\mathbf{T}_{8}$ & $\begin{array}{l}\text { Chlorantraniliprole } 18.5 \% \mathrm{SC} \\
\text { + Isoprothiolane } 40 \% \mathrm{EC}\end{array}$ & $0.3+1.5$ & $\begin{array}{l}18.96 \\
(25.73)\end{array}$ & $\begin{array}{l}18.37^{\mathrm{cd}} \\
(25.35)\end{array}$ & $\begin{array}{l}16.70^{\mathrm{cd}} \\
(22.12)\end{array}$ & $\begin{array}{l}13.86^{\mathrm{b}} \\
(21.76)\end{array}$ & $\begin{array}{l}16.31^{b} \\
(22.07)\end{array}$ & 63.80 \\
\hline$T_{9}$ & Untreated check & - & $\begin{array}{l}18.37 \\
(25.33)\end{array}$ & $\begin{array}{l}36.14^{\mathrm{a}} \\
(36.96)\end{array}$ & $\begin{array}{l}41.92^{a} \\
(40.36)\end{array}$ & $\begin{array}{l}58.81^{a} \\
(50.12)\end{array}$ & $\begin{array}{l}45.62^{a} \\
(42.48)\end{array}$ & 0 \\
\hline & F test & & NS & Sig. & Sig. & Sig. & Sig. & \\
\hline & $\mathrm{SEm} \pm$ & & 1.281 & 1.404 & 1.458 & 1.456 & 1.928 & \\
\hline & $\mathrm{CD}(\mathrm{P}=0.05)$ & & - & 4.211 & 4.373 & 4.365 & 8.48 & \\
\hline & $\mathrm{CV}(\%)$ & & 13.36 & 12.27 & 13.58 & 14.00 & 16.63 & \\
\hline
\end{tabular}

*DBS- day before spray **DAS- days after spray

***Figures in parenthesis are Arc-sine transformed values

Means followed by same letter do not differ significantly following DMRT 
At 30 days after spraying (DAS), tricyclazole $\left(0.6 \mathrm{~g} \mathrm{l}^{-1}\right)$ emerged as the best treatment by recording lowest (7.70) per cent leaf blast incidence which was on par with isoprothiolane $\left(1.5 \mathrm{ml} \mathrm{l}^{-1}\right)$ with $10.59 \%$ leaf blast incidence followed by chlorantraniliprole + tricyclazole $\left(0.3 \mathrm{ml} \mathrm{l}^{-1}+\right.$ $0.6 \mathrm{~g} \mathrm{l}^{-1}$ ) with $10.61 \%$ leaf blast incidence, chlorpyriphos + isoprothiolane $\left(2.5 \mathrm{ml} \mathrm{l}^{-1}+1.5\right.$ $\mathrm{ml} \mathrm{l}^{-1}$ ) with $11.70 \%$ leaf blast incidence. However, untreated check has recorded the highest per cent leaf blast incidence of $58.81 \%$.

The mean data of per cent blast incidence recorded on 10, 20 and 30 days after spraying revealed that tricyclazole $\left(\begin{array}{llll}0.6 & \mathrm{~g} \mathrm{l}^{-1}\end{array}\right)$ was superior over other treatments by recording 11.8 per cent blast incidence with 74.13 per cent disease incidence reduction over control. These results are in accordance with Dutta et al., (2012) who reported that tricyclazole $75 \%$ WP proved effective in management of rice blast disease with $12.85 \%$ leaf blast incidence.

Isoprothiolane $\left(1.5 \mathrm{ml} \mathrm{l}^{-1}\right)$ recorded 13.95 mean per cent blast incidence and was on par with the best treatment. These results are in acceptance with Singh et al., (2011) who reported that isoprothiolane @ $1.5 \mathrm{ml} \mathrm{l}^{-1}$ was effective after tricyclazole @ $0.6 \mathrm{~g} \mathrm{l}^{-1}$ in controlling blast incidence with 22.6 and 17.6 $\%$ neck blast incidence in the year 2008 and 2009, respectively.

The treatments chlorantraniliprole + isoprothiolane $\left(0.3 \mathrm{ml} \mathrm{l}^{-1}+1.5 \mathrm{ml} \mathrm{l} \mathrm{l}^{-1}\right)$, chlorantraniliprole + tricyclazole $\left(0.3 \mathrm{ml} \mathrm{l}^{-}\right.$ $\left.{ }^{1}+0.6 \mathrm{~g} \mathrm{l}^{-1}\right)$, chlorpyriphos + isoprothiolane $\left(2.5 \mathrm{ml} \mathrm{l}^{-1}+1.5 \mathrm{ml} \mathrm{l}^{-1}\right)$ and chlorpyriphos + tricyclazole $\left(2.5 \mathrm{ml} \mathrm{l}^{-1}+0.6 \mathrm{~g} \mathrm{l}^{-1}\right)$ followed the best treatment by recording 16.31, 17.64, 17.72 and 19.45 mean per cent blast incidence and $63.80,61.33,61.15$ and 57.36 per cent disease incidence reduction over control, respectively. These results are in accordance Kumar et al., (2011) reported that isoprothiolane $1.5 \mathrm{ml} \mathrm{l}^{-1}+$ flubendamide $0.5 \mathrm{~g}$ $1^{-1}$ recorded $20 \%$ leaf blast severity compared to isoprothiolane alone treatment with $24 \%$ leaf blast severity.

From the above findings it is clear that all fungicidal treatments are almost similar in their bioefficacy in controlling the blast disease when used alone or in combination with insecticides. All individual fungicidal treatments were statistically on par with themselves and also with combination treatments and there was no significant difference between individual treatments and when combined with insecticides implying compatibility between the test insecticides and fungicides in their bioefficacy in controlling leaf blast disease.

\section{Effect of treatments on yield}

Significant difference between the treatments was observed with respect to yield. The yield ranged from $2.00 \mathrm{t} \mathrm{ha}^{-1}$ to $5.39 \mathrm{t} \mathrm{ha}^{-1}$ (Table 6 and Fig. 5).

Significantly highest $\left(5.39 \mathrm{t} \mathrm{ha}^{-1}\right)$ yield was recorded in chlorantraniliprole + isoprothiolane $\left(0.3 \mathrm{ml} \mathrm{l}^{-1}+1.5 \mathrm{ml} \mathrm{l}^{-1}\right)$ which was on par with all the other insecticide and fungicide combinations tested in the present investigation i.e., chlorantraniliprole +

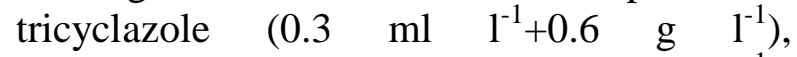
chlorpyriphos + isoprothiolane $\left(2.5 \mathrm{ml} \mathrm{l}^{-1}+\right.$ $1.5 \mathrm{ml} \mathrm{l}^{-1}$ ) and chlorpyriphos + tricyclazole $\left(2.5 \mathrm{ml} \mathrm{l}^{-1}+0.6 \mathrm{~g} \mathrm{l}^{-1}\right)$ which recorded 5.07, 4.85 and $4.77 \mathrm{t} \mathrm{ha}^{-1}$, respectively. These results in accordance with Bhuvaneswari and Krishnamraju (2013), who reported that chlorantraniliprole @ $0.3 \mathrm{ml} / 1$ in combination with hexaconazole @ $2 \mathrm{ml} \mathrm{l}^{-1}$ gave less incidence $(8.3 \%)$ and severity of sheath blight $(12.8 \%)$ and also less stem borer and lesser leaf folder and recorded $5.6 \mathrm{t} \mathrm{ha}^{-1}$ of grain 
yield of rice. Similarly findings of Kumar, (2011) revealed that isoprothiolane + fipronil, isoprothiolane + flubendamide, tricyclazole + fipronil and tricyclazole + flubendamide gave satisfactory yields of 4.97, 5.19, 5.14 and 5.17 t/ha, respectively whereas Singh et al., (2011) reported that Isoprothiolane + fenoxanil yielded $3.8 \mathrm{t} \mathrm{ha}^{-1}$.

\section{References}

Bhuvaneswari, V and Krishnamraju, S. 2013. Compatibility of fungicides and insecticides targeting sheath blight and major rice pests. Journal of Rice Research. 6 (2): 15-18.

Dutta, D., Saha, S., Ray, D.P and Bag, M.K. 2012. Effect of different active fungicide molecules on the management of rice blast disease. International Journal of Agriculture, Environment and Biotechnology. 5 (3): 247-251.

Kumar, P.M.K., Sidde Gowda, D.K., Gowda, K.T.P., Arpita, K.S and Bhat, G. 2011. Compatibility and efficacy of insecticides and fungicide mixtures against major pests and diseases in rice. Pestology. 35: 17-21.

Kushwana, K.S and Singh, R. 1984. Leaf folder (Cnaphalocrocis medinalis) outbreak in Haryana. International Rice Research Newsletter. 9 (6): 20.

Prajapati, K.S., Patel, R.C and Pathak, A.R. 2004. Field evaluation of new fungicides against blast of rice. Pesticide Research Journal. 16 (2): 2628.

Siddegowda, D.K. 2009. Field evaluation of Virtako $40 \%$ WG (chlorantraniliprole + thiamethoxam) against rice pest complex. Pestology. 33 (4): 32-36.

Singh, R., Sunder, S., Dodan, D.S and Ram, L. 2011. Sources of resistance to blast and its management through chemicals. Journal of Mycology and Plant Pathology. 41 (3): 422-425.

Suri, K.S. 2011. Bioefficacy of some insecticides against rice stem borers and leaf folder infesting rice in Punjab. Pesticide Research Journal. 23 (2): 190-193.

\section{How to cite this article:}

Anil Kumar, K., A.S.R. Sarma, T. Madhumathi and Prasanna Kumari, V. 2018. Study on Efficacy of Pesticide Mixtures as Foliar Sprays for the Management of Rice Leaf Folder (Cnaphalocrocis medinalis) and Leaf Blast (Pyricularia oryzae) on Paddy. Int.J.Curr.Microbiol.App.Sci. 7(11): 2163-2175. doi: https://doi.org/10.20546/ijcmas.2018.711.243 